\title{
The Role of High Flow Nasal Cannula in COVID-19 Associated Pneumomediastinum and Pneumothorax
}

\author{
Francesca Simioli * (D), Anna Annunziata, Giorgio Emanuele Polistina (D), Antonietta Coppola, Valentina Di Spirito \\ and Giuseppe Fiorentino
}

check for updates

Citation: Simioli, F.; Annunziata, A.; Polistina, G.E.; Coppola, A.; Di

Spirito, V.; Fiorentino, G. The Role of

High Flow Nasal Cannula in

COVID-19 Associated

Pneumomediastinum and

Pneumothorax. Healthcare 2021, 9, 620.

https://doi.org/10.3390/

healthcare 9060620

Academic Editor: Paolo Cotogni

Received: 22 April 2021

Accepted: 21 May 2021

Published: 22 May 2021

Publisher's Note: MDPI stays neutral with regard to jurisdictional claims in published maps and institutional affiliations.

Copyright: (C) 2021 by the authors. Licensee MDPI, Basel, Switzerland. This article is an open access article distributed under the terms and conditions of the Creative Commons Attribution (CC BY) license (https:// creativecommons.org/licenses/by/ $4.0 /)$.
Sub-intensive Care Unit, Department of Respiratory Pathophysiology and Rehabilitation Monaldi-A.O. Dei Colli, Via Gaetano Quagliariello 54, 80131 Naples, Italy; anna.annunziata@gmail.com (A.A.); giorgiopolistina@gmail.com (G.E.P.); antonietta.coppola84@gmail.com (A.C.); valentinadispirito@hotmail.com (V.D.S.); giuseppefiorentino1@gmail.com (G.F.)

* Correspondence: francesimioli@gmail.com

Abstract: Background: Pneumomediastinum, subcutaneous emphysema and pneumothorax are not rarely observed during the COVID-19 pandemic. Such complications can worsen gas exchange and the overall prognosis in critical patients. The aim of this study is to investigate what predisposing factors are related to pneumomediastinum and pneumothorax in SARS-CoV2-Acute Respiratory Distress Syndrome (ARDS), what symptoms may predict a severe and potentially fatal complication and what therapeutical approach may provide a better outcome. Methods: In this single center cohort study, we recorded data from 45 critically ill COVID-19 patients who developed one or more complicating events among pneumomediastinum, subcutaneous emphysema and pneumothorax. All patients showed ARDS and underwent non-invasive ventilation (NIV) at baseline. Patients with mild to moderate ARDS and pneumomediastinum/pneumothorax $(n=25)$ received High Flow Nasal Cannula (HFNC), while patients with severe ARDS and pneumomediastinum/pneumothorax underwent HFNC $(n=10)$ or invasive mechanical ventilation (IMV) $(n=10)$. Results: Pneumomediastinum/pneumothorax developed in $10.5 \%$ of subjects affected by SARS-coV2-ARDS. Dyspnea affected $40 \%$ and cough affected $37 \%$ of subjects. High resolution computed tomography of the chest showed bilateral diffuse ground glass opacities (GGO) in $100 \%$ of subjects. Traction bronchiolectasis, reticulation, crazy paving and distortion were observed in $64 \%$. Furthermore, 36\% showed subcutaneous emphysema. Non-severe ARDS cases received HFNC, and 76\% patients recovered from pneumomediastinum/pneumothorax over a median follow up of 5 days. Among severe ARDS cases the recovery rate of pneumomediastinum/pneumothorax was 70\% with the HFNC approach, and $10 \%$ with IMV. Conclusion: HFNC is a safe and effective ventilatory approach for critical COVID-19 and has a positive role in associated complications such as pneumomediastinum and pneumothorax.

Keywords: critical COVID-19; non-invasive ventilation; mechanical ventilation; ARDS; P-SILI

\section{Background}

COVID-19 is an emerging infectious disease caused by a novel coronavirus named SARS-CoV2. As the global pandemic progresses, novel presentations and uncommon complications are recognized [1]. Multiple case reports are available about spontaneous or iatrogenic pneumomediastinum and pneumothorax related to SARS-CoV2 [2-4]. Which factors are underlying these conditions remain unclear. Lastly, no data are available about what approach can ameliorate the outcome of these patients.

High Flow Nasal Cannula (HFNC) is a ventilatory support able to deliver a high flow of properly heated and humidified air. It provides a reliable oxygen dispatch and allows an elevated fraction of inhaled oxygen (FiO2), from $21 \%$ to virtually $100 \%$, by preventing the dilution of oxygen with room air. The relatively small interface and the physiological temperature of this therapy is responsible for a very high tolerance. Overall compliance is greater than non-invasive ventilation (NIV). HFNC demonstrated to improve 
oxygenation, work of breathing, respiratory rate and dyspnea scores in patients with hypoxemic respiratory failure [5]. Augmented work of breathing can be estimated based on respiratory rate and impaired thoraco-abdominal synchrony. These are signals of an acute respiratory distress that may lead to invasive or non-invasive ventilation. Lee et al. reported how HFNC improves signs of a distressed breathing and improves oxygenation. The authors conclude that HFNC reduces the need for endotracheal intubation, thus no evidence supports an effect on mortality.

During this COVID-19 pandemic, HFNC was widely used to support respiratory failure in critically affected patients, though there are no clinical trials clarifying its safety and efficacy [6]. Some concerns arose about initiating HFNC in patients affected by acute respiratory failure since prolonged hypoxemia may lead to worse outcomes, and, besides, it may delay the escalation to ventilation [7]. The adoption of HFNC as a primary or rescue therapy for respiratory failure secondary to COVID-19 has led to similar concerns [8]. As hypoxemic normocapnic respiratory failure is widely accepted to be an indicator for HFNC use, its use has been extensive for COVID-19 since the first step of the current pandemic. Pneumomediastinum and pneumothorax are not currently indicators for HFNC use. Limited data are reported about the consequences of using HFNC in subjects with concomitant pneumothorax. Baudin et al. described 177 HFNC episodes involving 145 subjects. Among this population, six preexisting pneumothoraces $(3 \%)$ were identified before HFNC initiation, none of which worsened under HFNC. Two episodes $(1 \%)$ of new pneumothoraces occurred [9].

\section{Aims and Scope}

The aim of this study is to investigate what predisposing factors are related to pneumomediastinum and pneumothorax in SARS-CoV2-ARDS, what symptoms may predict a severe and potentially fatal complication and what therapeutical approach may provide a better outcome.

\section{Methods}

This is a single center cohort study about use of HFNC in complicated COVID-19. It included 45 patients selected among those admitted to our department from August 2020 to January 2021 for critical COVID-19. Inclusion criteria were: SARS-CoV-2 infection confirmed by reverse-transcriptase-polymerase-chain-reaction assay, interstitial pneumonia assessed by a high-resolution computed tomography (HRCT) of the chest and acute respiratory distress syndrome (ARDS). In addition, patients were required to develop a complicating event such as pneumomediastinum and/or pneumothorax. All patients provided informed consent. All patients received the pharmacological standard of care, and a ventilatory support. Continuous positive airway pressure (CPAP) or HFNC were used at baseline according to disease severity.

The HRCT allowed to describe the presence of abnormalities and define a total severity score (TSS) according to Chung, as the sum of the extension of acute lung inflammatory lesions in each of the five lobes, with a range from 0 to 20. The HRCT was performed again to follow up the complications. The follow-up was to continue until discharge or death of all subjects. Patients presenting a non-severe ARDS with pneumomediastinum and/or pneumothorax underwent HFNC therapy, while severe ARDS cases underwent HFNC or invasive mechanical ventilation (IMV) based on SpO2, respiratory rate and respiratory muscle fatigue.

The study was approved by the local ethics committee of University of Campania "Luigi Vanvitelli" and A.O. dei Colli in accordance with the 1976 Declaration of Helsinki and its later amendments. All subjects consented to participate.

Results are reported as number and percentage for categorical variables and median and interquartile range (IQR) for continuous variables. The baseline characteristics reported in Table 1 were compared with a $t$-test. The probability of a severe ARDS with pneumomediastinum in presence of symptoms or not, and subcutaneous emphysema or not was 
tested by an odds ratio. The recovery rate from the complicating event between HFNC and IMV was tested by a two-tailed Fisher's exact test. A $p$-value $<0.05$ was considered statistically significant.

Table 1. Baseline characteristics.

\begin{tabular}{|c|c|c|c|}
\hline & Overall Cohort & $\begin{array}{l}\text { Mild-Moderate ARDS and Pneu- } \\
\text { momediastinum/Pneumothorax }\end{array}$ & $\begin{array}{l}\text { Severe ARDS and Pneumo- } \\
\text { mediastinum/Pneumothorax }\end{array}$ \\
\hline $\begin{array}{l}\text { Total } \\
\text { Number }\end{array}$ & 45 & 25 & 20 \\
\hline $\begin{array}{c}\text { Age } \\
\text { Median (IQR) }\end{array}$ & $68(58-72)$ & $68(59-74)$ & $63(57-70)$ \\
\hline $\begin{array}{l}\text { Emphysema } \\
\text { Number }\end{array}$ & 9 & 8 & 1 \\
\hline $\begin{array}{l}\text { Total severity score } \\
\text { Median (IQR) } \\
\end{array}$ & $16(12-18)$ & $14(12-16)$ & $16(15.5-18)$ \\
\hline $\begin{array}{l}\text { Ground glass opacities } \\
\text { Number (Percentage) }\end{array}$ & $45(100 \%)$ & $25(100 \%)$ & $20(100 \%)$ \\
\hline $\begin{array}{l}\text { Distortion/traction } \\
\text { Number (Percentage) }\end{array}$ & $29(64 \%)$ & $17(68 \%)$ & $12(60 \%)$ \\
\hline $\begin{array}{c}\text { Consolidations } \\
\text { Number (Percentage) }\end{array}$ & $11(24 \%)$ & $5(20 \%)$ & $6(30 \%)$ \\
\hline
\end{tabular}

\section{Results}

Pneumomediastinum/pneumothorax developed in $10.5 \%$ of subjects affected by SARS-CoV2-ARDS. Out of 45, 43 patients were male. Median age was 68 years. Of these, 9 subjects had a BMI $>30,9$ subjects showed emphysema at baseline and 1 subject had a previous allergic asthma diagnosis. Common symptoms at COVID-19 onset were fever $(82 \%)$, dyspnea $(40 \%)$ and cough $(37 \%)$. A 59-years old male experienced cough and chest pain, the CT scan showed bilateral pneumothorax at onset of the disease. Moreover, a 66 years-old female showed subcutaneous emphysema and pneumomediastinum at onset. None of the 2 were receiving oxygen or ventilation at home.

HRCT of the chest showed diffuse bilateral ground glass opacities (GGO) in all patients (Figure 1). Reticulation (Figure 2), traction bronchiectasis/bronchiolectasis (Figure 3), crazy paving and distortion (Figure 4) were observed in 29 patients (64\%) at baseline. The total severity score was significantly higher at baseline in patients who eventually developed severe versus non-severe ARDS and pneumomediastinum/pneumothorax (16 versus 14; $95 \%$ CI 0.42 to $3.88 ; p=0.01$ ), as reported in Table 1 . No other significant difference was observed at baseline.

At hospital admission all subjects needed ventilatory support. As a first choice, they underwent non-invasive ventilation. As a result, 27 patients received continuous positive airway pressure (CPAP) ventilation via oronasal or total face mask, 11 patients received helmet CPAP and 7 had HFNC at baseline, as reported in Table 2. 
Table 2. Type of ventilation and symptoms at onset of pneumomediastinum/pneumothorax.

\begin{tabular}{cccc}
\hline & $\begin{array}{c}\text { Overall Cohort } \\
\text { (Number) }\end{array}$ & $\begin{array}{c}\text { Mild-Moderate ARDS and Pneu- } \\
\text { momediastinum/Pneumothorax } \\
\text { (Number) }\end{array}$ & $\begin{array}{c}\text { Severe ARDS and Pneumo- } \\
\text { mediastinum/Pneumothorax } \\
\text { (Number) }\end{array}$ \\
\hline Mask CPAP & 27 & 15 & 12 \\
\hline Helmet CPAP & 11 & 5 & 6 \\
\hline HFNC & 7 & 6 & 1 \\
\hline $\begin{array}{c}\text { Duration of NIV before complication } \\
\text { (median days and IQR) }\end{array}$ & $8(5-12)$ & $8.5(6-13)$ & $7(5-10)$ \\
\hline Symptoms & 28 & 14 & 14 \\
\hline Subcutaneous emphysema & 16 & 5 & 11 \\
\hline No symptoms & 17 & 11 & 6 \\
\hline
\end{tabular}

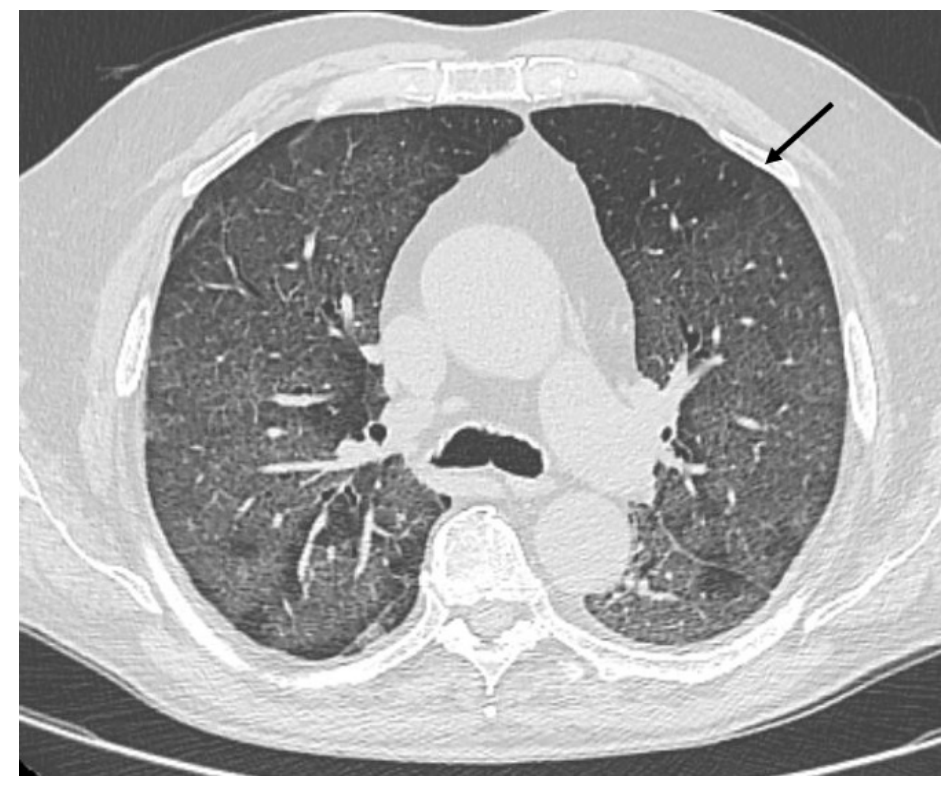

Figure 1. Diffuse bilateral ground glass opacities. Uneven distribution in the upper left lobe (arrow).

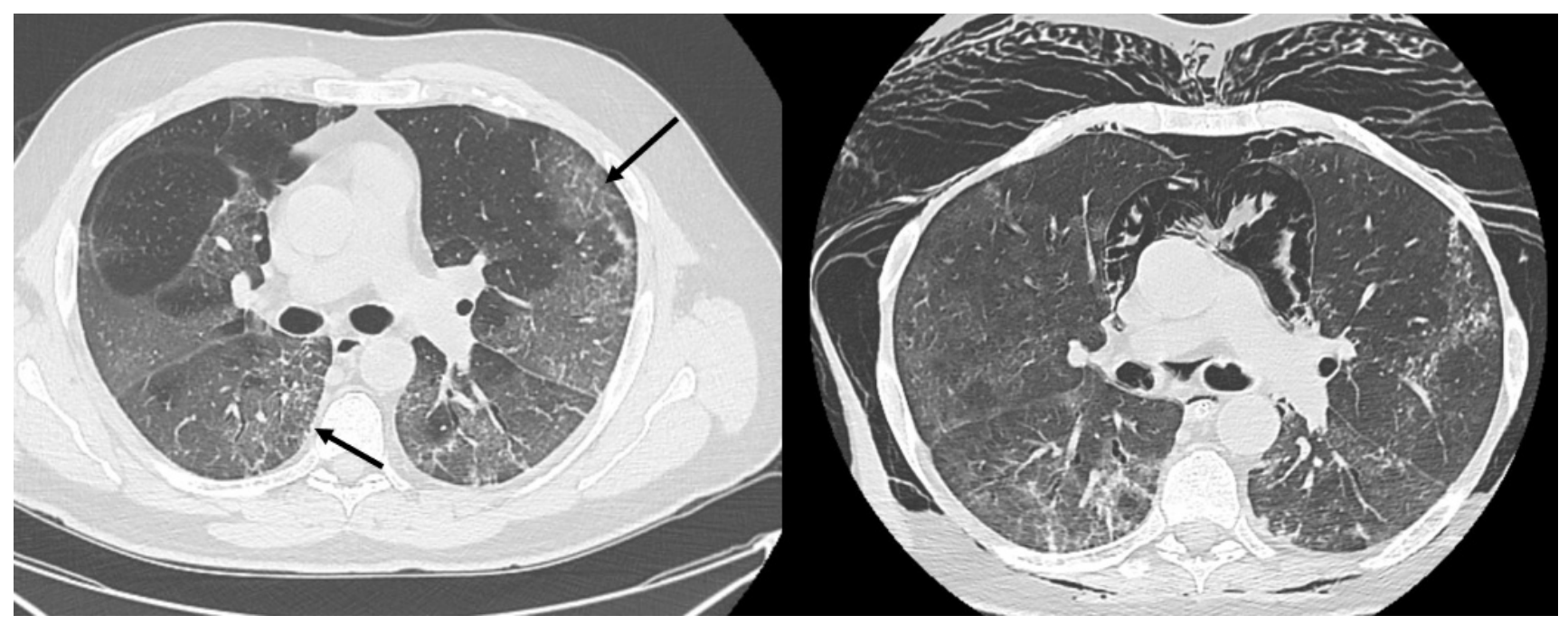

Figure 2. Reticular pattern (arrows) at baseline and massive pneumomediastinum after non-invasive ventilation in a 60-year-old man. 


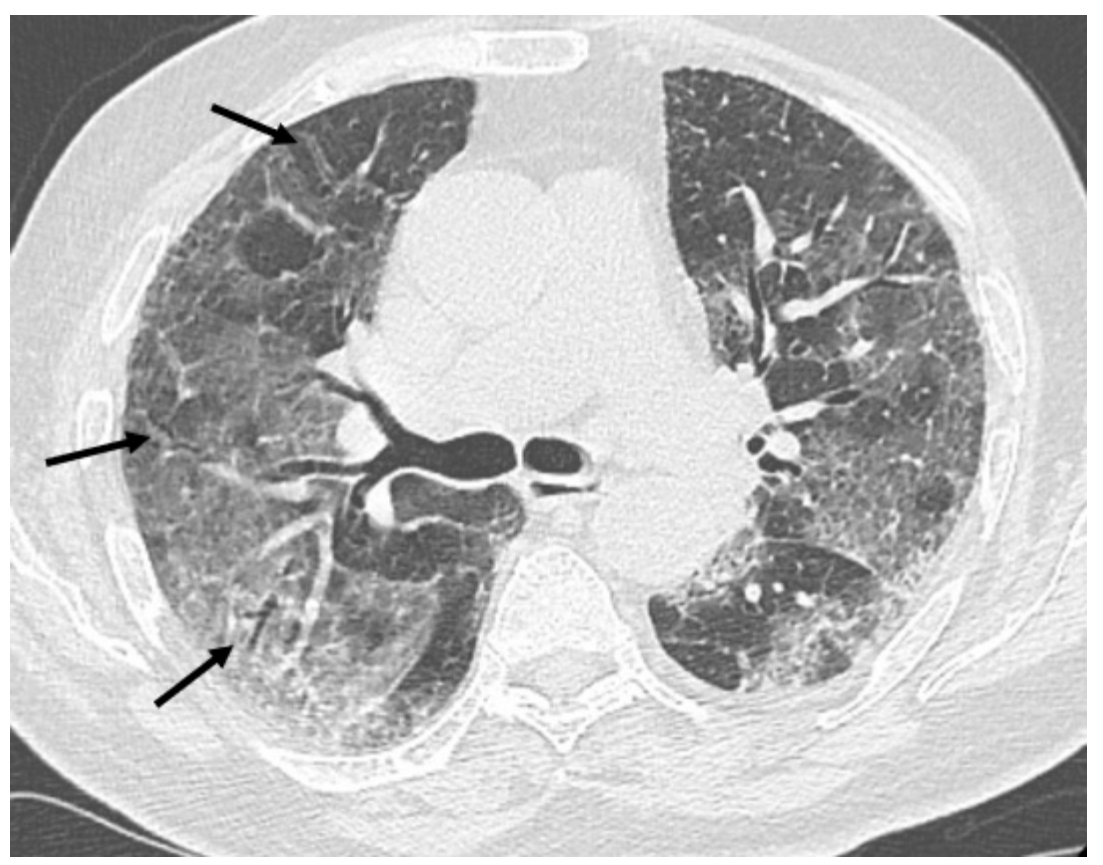

Figure 3. Bronchiolectasis (arrows) in the right lung.

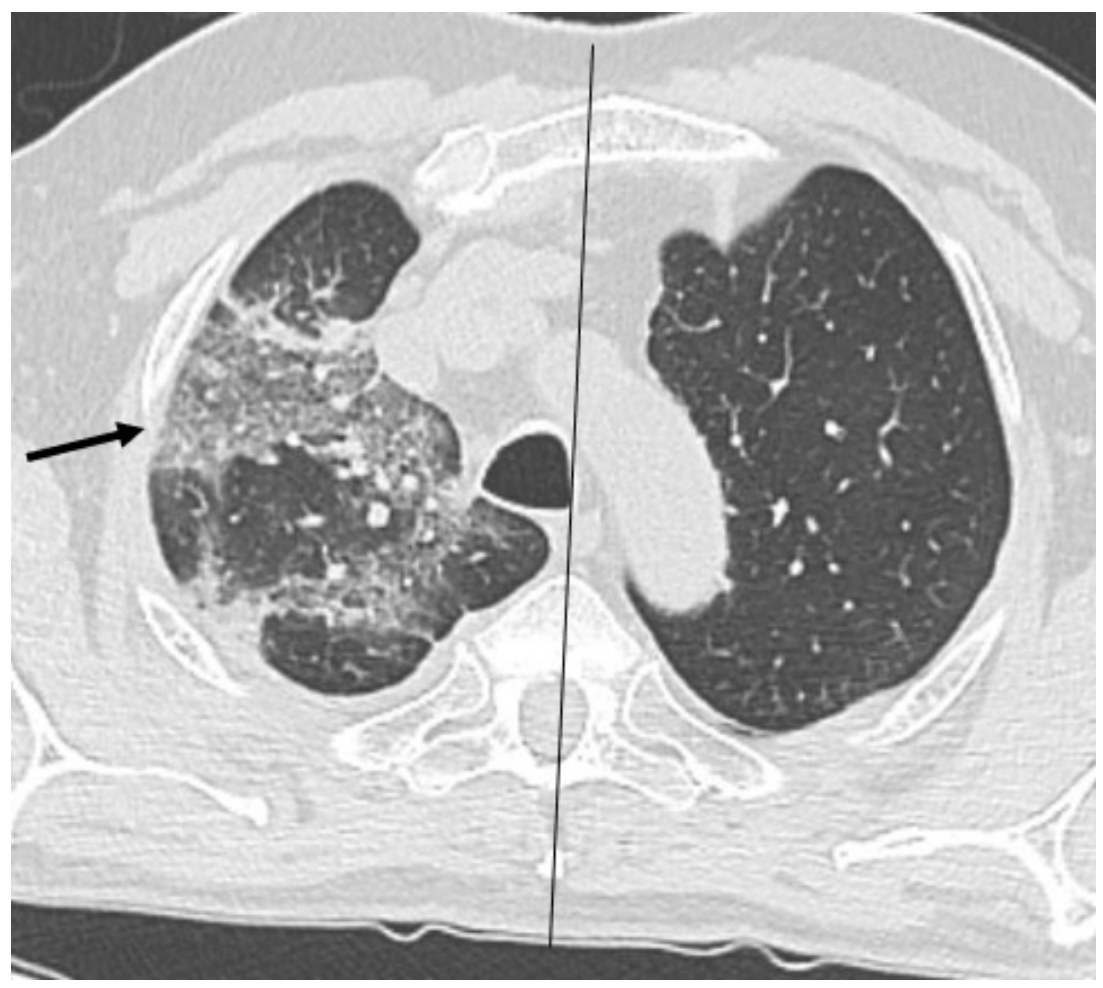

Figure 4. Crazy paving (arrow) and loss of volume of the upper right lobe.

The most common complication was pneumomediastinum. It occurred alone in 25 subjects; in 16 cases it was associated with pneumothorax. Pneumothorax alone was observed in 4 cases. In 28 cases (62\%), the diagnosis of pneumomediastinum and/or pneumothorax was suspected based on clinical conditions. Moreover, 16 subjects (36\%) showed subcutaneous emphysema. Additionally, 3 subjects experienced sinus tachycardia, and 3 had a supraventricular tachyarrhythmia. Chest pain was only referred in 4 cases. Finally, 17 patients were completely asymptomatic. The presence of symptoms 
is not significantly associated with a severe versus non-severe ARDS and pneumomediastinum/pneumothorax with an odds ratio of 1.83 (95\% CI: 0.53 to $6.33 ; p=0.33$ ). On the other hand, subcutaneous emphysema suggests a higher risk of a severe complication with an odds ratio of 4.89 (95\% CI 1.31 to $18.26 ; p=0.01)$.

Non-severe cases $(n=25)$ received HFNC set on $34^{\circ}$ Celsius and a flow range from 40 to $60 \mathrm{~L} / \mathrm{min}$. The fraction of inhaled oxygen $(\mathrm{FiO} 2)$ ranged between $55 \%$ and $100 \%$ to maintain a $\mathrm{SpO} 2 \geq 93 \%$. As a result, 19 patients recovered from pneumomediastinum/pneumothorax over a median follow up of 5 days. While 6 patients $(24 \%)$ died.

Severe cases received HFNC $(n=10)$ or IMV $(n=10)$. HFNC flow was set between 40-60 L/min and $\mathrm{FiO} 2$ ranged between $75-100 \%$. IMV was set on bilevel mode with a FiO2 range between $70-100 \%$. Among HFNC group, 7 patients recovered from the complicating event. Among IMV group, only 1 subject recovered from pneumomediastinum.

\section{Discussion}

The Macklin phenomenon explains how a great pressure gradient between alveoli and interstitium leads to air leak in the thin bronchovascular sheath causing pulmonary interstitial emphysema and ultimately pneumomediastinum. This circumstance can originate from an increase of intrathoracic pressure, as observed during cough or Valsalva maneuver. It reflects on intra-alveolar pressure and overdistension with rupture of alveoli. The resulting air leaks through interstitium towards a lower pressure part of the chest such as mediastinum. Mechanical ventilation is responsible for a higher alveolar end-expiratory pressure which can over-distend alveoli and decrease the venous caliber resulting in a ventilation-perfusion mismatch.

Low tidal volume lung protective ventilation is a cornerstone of supportive therapy in patients with ARDS, resulting in a significant improvement of the outcomes likely through mitigating the risk of induced lung injury (VILI). In spontaneously breathing patients with lung damage and reduced gas exchange, abnormal respiratory drive with large tidal volumes can exacerbate lung injury in a similar way, termed patient self-inflicted lung injury (P-SILI) [10]. The pathophysiologic basis has been postulated to be the result of a large fluctuation of transpulmonary pressure $\left(\mathrm{P}_{\mathrm{L}}\right)$ causing the delivery of quite high tidal volumes and overdistention of the smaller aerated and larger dependent atelectatic lung compartments [11]. Brisk diaphragm contraction may lead to intense local variations in $P_{L}$ predominantly affecting dependent lung, leading to additional stress with the drawing in of gas in from other non-dependent regions (pendelluft phenomenon) and compression with exhalation (atelectrauma) [12]. Additionally, pulmonary capillary vascular pressure and blood flow is augmented from large deflections of $P_{L}$ favoring the formation of pulmonary edema in injured lung units [13]. The harms of dysregulated high respiratory drive thus may lead to a cycle of continued lung injury, respiratory failure and possibly worse outcomes; potentially mitigated by early intubation.

Although intriguing and plausible, there is currently no prospective trial that offers conclusive evidence to this theory. Precise clinical measures defining P-SILI are also not established, but it still remains an important clinical construct. In an experimental model with spontaneously breathing animals, P-SILI was avoided with the application of a high PEEP; which led to recruitment of dependent lung regions, reduction of big $\mathrm{P}_{\mathrm{L}}$ changes and decreased inflammation seen on positron emission tomography [14].

Based on that, it is uncertain whether HFNC therapy in patients at risk for P-SILI is helpful or harmful. Nevertheless, we described two cases of pneumomediastinum at onset of disease, in non-ventilated patients, thus suggesting that other mechanisms may predispose COVID-19 patients to certain complicating events. Furthermore, seven cases occurred on HFNC which is an open system delivering quite low positive end-expiratory pressure (PEEP) [15].

The majority of our patients had no smoking history and emphysema affected only nine subjects. Cough was referred by $37 \%$ of subjects and can aggravate the risk of potentially negative outcome. Since then, further investigation is required to assess the 
indication for anti-tussive and sedatives being routinely used in awake patients to control cough. Laxatives should also be tested in order to avoid prolonged Valsalva maneuver.

From a clinical point of view, ARDS is typically characterized by an increased respiratory flow demand. The rationale for use of HFNC in acute hypoxemic respiratory failure is to reach this demand and reduce work of breathing. This scope is crucial in critical COVID-19, especially when complicating events modify ventilatory mechanics. In fact, in awake patients with ARDS, a ventilatory pattern with large tidal volumes causes major fluctuations of transpulmonary pressure leading to overdistension of alveoli. It has been postulated to exacerbate a patient self-inflicted lung injury (P-SILI). On the contrary HFNC has been shown to create maximal expiratory nasopharyngeal pressures of $5 \mathrm{~cm} \mathrm{H} 20$ at $50 \mathrm{~L} / \mathrm{M}$ flow rates, that are unlikely to be sufficient to recruit dependent lung regions [16]. Moreover, the same authors stated that HFNC delivers a variable pressure during the respiratory cycle with a maximum in early expiration. This mechanism supplies a PEEP thus promoting alveolar recruitment but also avoids alveolar overdistension even with a high flow. At the same time, in a small, randomized cross-over study the respiratory mechanics of HFNC at various flow rates were compared to facemask in subjects with hypoxemic respiratory failure. A flow of $60 \mathrm{~L} / \mathrm{M}$ demonstrated to significantly decrease transesophageal pressure fluctuations indicating patient effort and work of breathing [17]. This would suggest that in some patients HFNC may be adequate therapy to remit the risk of P-SILI.

Clinical measurements of work of breathing or respiratory drive including use of esophageal balloon manometry are cumbersome and impractical in spontaneously breathing patients with lung injury. Without clear guidance or endpoints, a decision to initiate or continue HFNC therapy to prevent lung injury rests on clinical judgment and gestalt. The incorporation of clinical scores predicting failure of HFNC may also be useful to discriminate in this setting.

It has been reported that pneumomediastinum in SARS-CoV2 ARDS may be related to lung frailty more than barotrauma [18]. In our population lung involvement assessed by HRCT revealed an extensive GGO. The median total severity score was $16 / 20$, so that in each lobe coexist pathological and normal groups of alveoli. This condition implies an uneven distribution of pressure during ventilation. In addition, frequently observed abnormalities include reticulation and bronchiolectasis which suggest a parenchymal distortion and possibly a reduction of lung compliance. Crazy paving is an interlobular and intralobular septal thickening, also common in ARDS. It is sometimes a cause of parenchymal distortion eventually leading to loss of lung volume and impaired compliance. We suggest that interstitial pneumonia can lead to architectural distortion and reduced compliance. This reflects on recruitability, in fact, in this condition lungs can tolerate a small variation or pressure, or driving pressure during non-invasive ventilation.

In this study we highlight that $74 \%$ of pneumomediastinum resolved after HFNC therapy. This observation suggests that HFNC is a safe ventilatory support for critical COVID-19 and has a potential role in associated complications. Despite this, its use remains controversial and further investigations are needed. The results of this study are limited by the small size cohort and the lack of a randomization.

\section{Conclusions}

HFNC is a safe and effective ventilatory support for critical COVID-19 and has a positive role in associated complications such as pneumomediastinum and pneumothorax. Antitussive and sedatives should be routinely prescribed to cure cough as well as to control abnormal ventilatory mechanics.

Author Contributions: Conceptualization, F.S.; Data curation, A.A.; Formal analysis, F.S.; Investigation, G.E.P. and A.C.; Methodology, F.S., A.A. and G.E.P.; Project administration, F.S.; Resources, G.E.P., A.C., V.D.S. and G.F.; Software, G.F.; Supervision, G.F.; Validation, A.C. and V.D.S.; Visualization, V.D.S.; Writing—original draft, F.S. and G.E.P.; Writing—review \& editing, A.A., A.C., V.D.S. and G.F. All authors have read and agreed to the published version of the manuscript. 
Funding: This research received no external funding.

Institutional Review Board Statement: Approved by the ethics committee of University of Campania "Luigi Vanvitelli" with number 2020/485 on 11/26/20.

Informed Consent Statement: All subjects provided informed consent.

Acknowledgments: All authors contributed and gave final approval of the version to be published. Other assistance with the article: none. Financial support and sponsorship: no financial support was received for this study.

Conflicts of Interest: The authors declare no conflict of interest.

\section{References}

1. Polistina, G.E.; Simioli, F.; Imitazione, P.; Lanza, M.; Annunziata, A.; Fiorentino, G. Different presentation of pulmonary parenchymal disruption in COVID-19 pneumonia. Case series of Sub-Intensive Care Unit in Naples, Italy. Monaldi Arch. Chest Dis. 2020, 90. [CrossRef] [PubMed]

2. Mohan, V.; Tauseen, R.A. Spontaneous pneumomediastinum in COVID-19. BMJ Case Rep. 2020, 13, e236519. [CrossRef] [PubMed]

3. Wang, W.; Gao, R.; Zheng, Y.; Jiang, L. COVID-19 with spontaneous pneumothorax, pneumomediastinum and subcutaneous emphysema. J. Travel Med. 2020, 27, taaa062. [CrossRef] [PubMed]

4. Goldman, N.; Ketheeswaran, B.; Wilson, H. COVID-19-associated pneumomediastinum. Clin. Med. 2020, 20, e91-e92. [CrossRef] [PubMed]

5. Lee, C.C.; Mankodi, D.; Shaharyar, S.; Ravindranathan, S.; Danckers, M.; Herscovici, P.; Moor, M.; Ferrer, G. High flow nasal cannula versus conventional oxygen therapy and non-invasive ventilation in adults with acute hypoxemic respiratory failure: A systematic review. Respir. Med. 2016, 121, 100-108. [CrossRef]

6. Suffredini, D.A.; Allison, M.G. A Rationale for Use of High Flow Nasal Cannula for Select Patients with Suspected or Confirmed Severe Acute Respiratory Syndrome Coronavirus-2 Infection. J. Intensive Care Med. 2021, 36, 9-17. [CrossRef] [PubMed]

7. Esteban, A.; Frutos-Vivar, F.; Ferguson, N.D.; Arabi, Y.; Apezteguía, C.; González, M.; Epstein, S.K.; Hill, N.S.; Nava, S.; Soares, M.A.; et al. Noninvasive positive-pressure ventilation for respiratory failure after extubation. N. Engl. J. Med. 2004, 350, 2452-2460. [CrossRef] [PubMed]

8. Simioli, F.; Annunziata, A.; Langella, G.; Polistina, G.E.; Martino, M.; Fiorentino, G. Clinical outcomes of high-flow nasal cannula in COVID-19 associated postextubation respiratory failure. A single-centre case series. Anaesthesiol. Intensive Ther. 2020, 52, 373-376. [CrossRef] [PubMed]

9. Baudin, F.; Gagnon, S.; Crulli, B.; Proulx, F.; Jouvet, P.; Emeriaud, G. Modalities and Complications Associated with the Use of High-Flow Nasal Cannula: Experience in a Pediatric ICU. Respir. Care 2016, 61, 1305-1310. [CrossRef] [PubMed]

10. Brochard, L.; Slutsky, A.; Pesenti, A. Mechanical ventilation to minimize progression of lung injury in acute respiratory failure. Am. J. Respir. Crit. Care Med. 2017, 195, 438-442. [CrossRef] [PubMed]

11. Grieco, D.L.; Menga, L.S.; Eleuteri, D.; Antonelli, M. Patient selfinflicted lung injury: Implications for acute hypoxemic respiratory failure and ARDS patients on non-invasive support. Minerva Anestesiol 2019, 85, 1014-1023. [CrossRef] [PubMed]

12. Yoshida, T.; Grieco, D.L.; Brochard, L.; Fujino, Y. Patient self-inflicted lung injury and positive end-expiratory pressure for safe spontaneous breathing. Curr. Opin. Crit. Care 2020, 26, 59-65. [CrossRef] [PubMed]

13. Yoshida, T.; Amato, M.B.; Kavanagh, B.P.; Fujino, Y. Impact of spontaneous breathing during mechanical ventilation in acute respiratory distress syndrome. Curr. Opin. Crit. Care 2019, 25, 192-198. [CrossRef]

14. Morais, C.C.; Koyama, Y.; Yoshida, T.; Plens, G.M.; Gomes, S.; Lima, C.A.; Ramos, O.P.; Pereira, S.M.; Kawaguchi, N.; Yamamoto, H.; et al. High positive end-expiratory pressure renders spontaneous effort noninjurious. Am. J. Respir. Crit. Care Med. 2018, 197, 1285-1296. [CrossRef] [PubMed]

15. Ritchie, J.E.; Williams, A.B.; Gerard, C.; Hockey, H. Evaluation of a humidified nasal high-flow oxygen system, using oxygraphy, capnography and measurement of upper airway pressures. Anaesth. Intensive Care 2011, 39, 1103-1110. [CrossRef] [PubMed]

16. Parke, R.L.; McGuinness, S.P. Pressures delivered by nasal high flow oxygen during all phases of the respiratory cycle. Respir. Care 2013, 58, 1621-1624. [CrossRef] [PubMed]

17. Mauri, T.; Alban, L.; Turrini, C.; Cambiaghi, B.; Carlesso, E.; Taccone, P.; Bottino, N.; Lissoni, A.; Spadaro, S.; Volta, C.A.; et al. Optimum support by high-flow nasal cannula in acute hypoxemic respiratory failure: Effects of increasing flow rates. Intensive Care Med. 2017, 43, 1453-1463. [CrossRef] [PubMed]

18. Lemmers, D.H.; Abu Hilal, M.; Bnà, C.; Prezioso, C.; Cavallo, E.; Nencini, N.; Crisci, S.; Fusina, F.; Natalini, G. Pneumomediastinum and subcutaneous emphysema in COVID-19: Barotrauma or lung frailty? ERJ Open Res. 2020, 6, 00385-2020. [CrossRef] [PubMed] 